TRANSACTIONS OF THE

AMERICAN MATHEMATICAL SOCIETY

Volume 363, Number 1, January 2011, Pages 345-363

S 0002-9947(2010)05153-0

Article electronically published on August 18, 2010

\title{
A CHARACTERIZATION AND A GENERALIZATION OF $W^{*}$-MODULES
}

\author{
DAVID P. BLECHER AND UPASANA KASHYAP
}

\begin{abstract}
We give a new Banach module characterization of $W^{*}$-modules, also known as self-dual Hilbert $C^{*}$-modules over a von Neumann algebra. This leads to a generalization of the notion, and the theory, of $W^{*}$-modules, to the setting where the operator algebras are $\sigma$-weakly closed algebras of operators on a Hilbert space. That is, we find the appropriate weak* topology variant of our earlier notion of rigged modules, and their theory, which in turn generalizes the notions of a $C^{*}$-module and a Hilbert space, successively. Our $w^{*}$-rigged modules have canonical 'envelopes' which are $W^{*}$-modules. Indeed, a $w^{*}$ rigged module may be defined to be a subspace of a $W^{*}$-module possessing certain properties.
\end{abstract}

\section{INTRODUCTION AND NOTATION}

A $W^{*}$-module is a Hilbert $C^{*}$-module over a von Neumann algebra which is selfdual, or, equivalently, which has a predual (see e.g. [23, 14, 11]). These objects were first studied by Paschke, and then by Rieffel [21, 22] (see also e.g. [10, Section 8.7] for an account of their theory). These objects are by now fundamental in $C^{*}$ algebra theory and noncommutative geometry, being intimately related to Connes' correspondences for example (see e.g. [1] for the relationship). $W^{*}$-modules have many characterizations; the one mentioned in our title characterizes them in the setting of Banach modules in a new way. This in turn leads us to generalize the notion of ' $W^{*}$-module' to the setting of modules over a dual operator algebra (by which we mean a $\sigma$-weakly closed algebra of operators on a Hilbert space). The new class of modules we call $w^{*}$-rigged modules. These are the appropriate weak* topology variant of our earlier notion of rigged modules (see e.g. 2, 12, 3, 6]), which in turn generalizes the notions of Hilbert $C^{*}$-modules and Hilbert space, successively. $W^{*}$-rigged modules will have an extensive theory; we will only present the basics here. Our main motivation for this project is that many ideas in the $W^{*}$ module theory, such as 'induced representations', are beautiful and fundamental, and thus should be important in a larger context. We also wished to enlarge the universe in which $W^{*}$-modules reside and act. In addition, one obtains many theorems reprising basic facts from the theory of rings and modules, but which only make sense for modules satisfying our definition (for example because direct sums are problematic for general operator modules).

Received by the editors December 7, 2007 and, in revised form, December 10, 2007 and March 23, 2009.

2010 Mathematics Subject Classification. Primary 46L08, 47L30, 47L45; Secondary 16D90, $47 \mathrm{~L} 25$.

The first author was supported by grant DMS 0400731 from the National Science Foundation. 
Our theory utilizes several pretty ideas from operator space theory. For example, our theory of the space of left multipliers $\mathcal{M}_{\ell}(X)$ of an operator space $X$ (see e.g. [10. Chapter 4]) plays a considerable role in our paper. Indeed the absence of this tool, and also of a recently introduced module tensor product [17] (see also [8, Section 2]) is the main reason why headway was not made on this project many years ago.

Unlike the $W^{*}$-module situation, $w^{*}$-rigged modules do not necessarily give rise to a weak* Morita equivalence in the sense of our earlier paper 8 . Nor are they all complemented submodules of a direct sum of copies of $M$, as is the case for $W^{*}$-modules 21. However, each $w^{*}$-rigged module has a canonical $W^{*}$-module envelope, called the $W^{*}$-dilation (and thus $w^{*}$-rigged modules give new examples of $W^{*}$-modules). This dilation is an important tool in our theory. Indeed, a $w^{*}$ rigged module may be defined to be a subspace of a $W^{*}$-module possessing certain properties. Thus there is a von Neumann algebra valued inner product (and Morita equivalence) around.

The material on $W^{*}$-modules in Section 2 is closely related to a paper of the first author from about ten years ago [5]. The main point of the latter paper was that $W^{*}$-modules fall comfortably into a dual operator module setting; for example, their usual tensor product (sometimes called 'composition of $W^{*}$-correspondences') agrees with a certain operator space tensor product studied by Magajna. This had certain advantages, for example new results about this tensor product (see also [13). Here we show for example that this tensor product also equals the normal module Haagerup tensor product recently introduced in [17] and studied further in [8. In Section 3 we find the variant for $w^{*}$-rigged modules of the basic theory of rigged modules from [2] (and to a lesser extent, [12]). In Section 4 we give several alternative equivalent definitions of $w^{*}$-rigged modules, some of which the reader may prefer. In Section 5 we give many examples of $w^{*}$-rigged modules.

Since a number of the ideas and proofs here are quite analogous to those from our papers on related topics, principally [2, 12, 8, 11, we will be quite brief in many of the proofs. We assume that the reader has some familiarity with these earlier ideas and proof techniques, and will often merely indicate the new techniques which allow one to modify arguments from 2, 12, to the present (weak* topology) setting. As the paper proceeds we will include fewer and fewer details, since we will be assuming that the reader is growing in familiarity with the methods introduced earlier and in 8 , and the ways these are used to modify proofs from the older theory. Also, a few complementary facts may be found in [19, 18]. Indeed the present paper represents a research program that the first author suggested to the second while she was a graduate student, and it was intended that full details and other aspects of the theory of $w^{*}$-rigged modules not touched upon here would be presented elsewhere. This project is still in progress.

We will use the notation of our previous paper [8]. We will assume that the reader is familiar with basic notions from operator space theory, as may be found in any of the current texts on that subject, and the application of this theory to operator algebras (see e.g. [10]). The latter source may also be consulted for background and for any unexplained terms below.

We also assume that the reader is familiar with basic Banach space (and operator space) duality principles (see e.g. [10, Sections 1.4, 1.6, Appendix A.2]). We will often abbreviate 'weak"' to ' $w^{*}$ '. Throughout the paper, $H$ and $K$ denote Hilbert 
spaces. Unless indicated otherwise, $M$ denotes a dual operator algebra, that is, an operator algebra which is also a dual operator space. We take all dual operator algebras $M$ to be unital; that is, we assume they possess an identity of norm 1 (this is for convenience; for nonunital algebras $M$ one may consider the unitization $M^{1}$ (see [10, 2.7.4 (5)]), for example defining a module over a nonunital algebra $M$ to be $w^{*}$-rigged if it is $w^{*}$-rigged over $M^{1}$ ). By well-known duality principles, any $w^{*}$-closed subalgebra of $B(H)$ is a dual operator algebra. Conversely, for any dual operator algebra $M$, there exists a Hilbert space $H$ and a $w^{*}$-continuous completely isometric homomorphism $\pi: M \rightarrow B(H)$ (see e.g. [10, Theorem 2.7.9]). Then the range $\pi(M)$ is a $w^{*}$-closed subalgebra of $B(H)$, which we may identify with $M$ in every way.

For cardinals or sets $I, J$, we use the symbol $M_{I, J}(X)$ for the operator space of $I \times J$ matrices over an operator space $X$, whose 'finite submatrices' have uniformly bounded norm. We set $C_{J}^{w}(X)=M_{J, 1}(X)$ and $R_{J}^{w}(X)=M_{1, J}(X)$, and these are written as $C_{n}(X)$ and $R_{n}(X)$ if $J=n$ is finite.

A concrete left operator module over an operator algebra $A$ is a linear subspace $X \subset B(K, H)$ such that $\pi(A) X \subset X$ for a completely contractive representation $\pi: A \rightarrow B(H)$. An abstract operator A-module is an operator space $X$ which is also an $A$-module, such that $X$ is completely isometrically isomorphic, via an $A$-module map, to a concrete operator $A$-module and similarly for right modules, or bimodules. Most of the interesting modules over operator algebras are operator modules, such as Hilbert $C^{*}$-modules (the operator space structure on a $C^{*}$-module is the one it receives as a subspace of its linking $C^{*}$-algebra; see e.g. 10, Section $8.2]$ ).

A normal Hilbert module over a dual operator algebra $M$ is a pair $(H, \pi)$, where $H$ is a (column) Hilbert space (see e.g. 1.2.23 in [10]), and $\pi: M \rightarrow B(H)$ is a $w^{*}$-continuous unital completely contractive representation. We shall call such a $\pi$ a normal representation of $M$. The module action is given by $m \cdot \zeta=\pi(m) \zeta$.

A concrete dual operator $M-N$-bimodule is a $w^{*}$-closed subspace $X$ of $B(K, H)$ such that $\theta(M) X \pi(N) \subset X$, where $\theta$ and $\pi$ are normal representations of $M$ and $N$ on $H$ and $K$ respectively. An abstract dual operator $M-N$-bimodule is defined to be a nondegenerate operator $M$ - $N$-bimodule $X$, which is also a dual operator space, such that the module actions are separately weak* continuous. Such spaces can be represented completely isometrically as concrete dual operator bimodules, and in fact this can be done under even weaker hypotheses (see e.g. [10, 11, 15]) and similarly for one-sided modules (the case $M$ or $N$ equals $\mathbb{C}$ ). We use standard notation for module mapping spaces; e.g. $C B(X, N)_{N}\left(\right.$ resp. $\left.w^{*} C B(X, N)_{N}\right)$ are the completely bounded (resp. and $w^{*}$-continuous) right $N$-module maps from $X$ to $N$. Any normal Hilbert $M$-module $H$ (with its column Hilbert space structure $H^{c}$ ) is a left dual operator $M$-module. In a couple of proofs, we will assume that the reader is familiar with the theory of multipliers of an operator space $X$ (see e.g. [10, Chapter 4]). We recall that the left multiplier algebra $\mathcal{M}_{\ell}(X)$ of $X$ is a collection of certain operators on $X$, which are weak* continuous if $X$ is a dual operator space [11. Indeed, in the latter case, $\mathcal{M}_{\ell}(X)$ is a dual operator algebra.

A bilinear map $u: X \times Y \rightarrow Z$ is $M$-balanced if $u(x m, y)=u(x, m y)$ for $m \in M$ and completely contractive if it corresponds to a linear complete contraction on $X \otimes_{h} Y$. We use the normal module Haagerup tensor product $\otimes_{M}^{\sigma h}$ throughout the paper, and its universal property from [17, which loosely says that it 'linearizes 
completely contractive $M$-balanced separately weak* continuous bilinear maps'. Every completely contractive separately weak* continuous $M$-balanced map $u$ : $X \times Y \rightarrow Z$ induces a completely contractive weak* continuous complete contraction $X \otimes_{M}^{\sigma h} Y \rightarrow Z$. We also assume that the reader is familiar with the notation and facts about $\otimes_{M}^{\sigma h}$ from [8, Section 2].

\section{2. $W^{*}$-MODULES}

We begin this section with a useful lemma:

Lemma 2.1. Let $\left\{H_{\alpha}\right\}$ be a collection of Hilbert spaces (resp. column Hilbert spaces) indexed by a directed set. Let $Y$ be a dual Banach space (resp. dual operator space). Suppose there exist $w^{*}$-continuous contractive (resp. completely contractive) linear maps $\phi_{\alpha}: Y \rightarrow H_{\alpha}, \psi_{\alpha}: H_{\alpha} \rightarrow Y$, such that $\psi_{\alpha}\left(\phi_{\alpha}(y)\right) \stackrel{w^{*}}{\rightarrow} y$ for each $y \in Y$. Then $Y$ is a Hilbert space (resp. column Hilbert space). The inner product on $Y$ is $\langle y, z\rangle=\lim _{\alpha}\left\langle\phi_{\alpha}(y), \phi_{\alpha}(z)\right\rangle$, for $y, z \in Y$.

Proof. The proof that $Y$ is a Hilbert space (resp. column Hilbert space) follows by the ultraproduct argument in Theorem 3.10 in $[8$. For the last assertion, we will show first that $\left\|\phi_{\alpha}(y)\right\|^{2} \rightarrow\|y\|^{2}$. Then, by the polarization identity, it follows that $\langle y, z\rangle=\lim _{\alpha}\left\langle\phi_{\alpha}(y), \phi_{\alpha}(z)\right\rangle$, as desired. Suppose there exists a subnet $\left(\phi_{\alpha_{t}}(y)\right)$ such that $\left\|\phi_{\alpha_{t}}(y)\right\|^{2} \rightarrow \beta$. We need to prove that $\beta=\|y\|^{2}$. Clearly $\beta \leq\|y\|^{2}$. If $\beta<K<\|y\|^{2}$, then there exists a $t_{0}$ such that $\left\|\phi_{\alpha_{t}}(y)\right\|^{2} \leq K$ for all $t \geq t_{0}$. This implies that $\left\|\psi_{\alpha_{t}} \phi_{\alpha_{t}}(y)\right\|^{2} \leq\left\|\phi_{\alpha_{t}}(y)\right\|^{2} \leq K$ for all $t \geq t_{0}$. Since $\psi_{\alpha_{t}} \phi_{\alpha_{t}}(y) \stackrel{w^{*}}{\rightarrow} y$, by Alaoglu's theorem we deduce that $\|y\|^{2} \leq K$, which is a contradiction.

We now generalize the notion of $W^{*}$-modules to the setting where the operator algebras are $\sigma$-weakly closed algebras of operators on a Hilbert space. The following is the 'weak* topology variant' of the notion of rigged module studied in 2, 12, 3, 6] (the last paper has the most succinct definition of these objects, and [3] is a survey). In Section 4 we will prove several equivalent, but quite different looking, characterizations of $w^{*}$-rigged modules.

Definition 2.2. Suppose that $Y$ is a dual operator space and a right module over a dual operator algebra $M$. Suppose that there exists a net of positive integers $(n(\alpha))$, and $w^{*}$-continuous completely contractive $M$-module maps $\phi_{\alpha}: Y \rightarrow C_{n(\alpha)}(M)$ and $\psi_{\alpha}: C_{n(\alpha)}(M) \rightarrow Y$, with $\psi_{\alpha}\left(\phi_{\alpha}(y)\right) \rightarrow y$ in the $w^{*}$-topology on $Y$, for all $y \in Y$. Then we say that $Y$ is a right $w^{*}$-rigged module over $M$.

It will require some nontrivial analysis to develop the theory of these modules from the definition given above, just as is the case in the norm topology variant, where one needs a deep theorem of Hay and other results [6]. We begin by noting that an argument similar to that in the last few lines of the proof of Lemma 2.1. and using basic operator space duality principles, shows that for a $w^{*}$-rigged module $Y$,

$$
\left\|\left[y_{i j}\right]\right\|_{M_{n}(Y)}=\sup _{\alpha}\left\|\left[\phi_{\alpha}\left(y_{i j}\right)\right]\right\|, \quad\left[y_{i j}\right] \in M_{n}(Y) .
$$

Theorem 2.3. If $Y$ is a right $w^{*}$-rigged module over a dual operator algebra $M$, then $w^{*} C B(Y)_{M}=\mathcal{M}_{\ell}(Y)$ completely isometrically isomorphically, and this is a weak*-closed subalgebra of $C B(Y)_{M}$. Hence $w^{*} C B(Y)_{M}$ is a dual operator algebra, and $Y$ is a left dual $w^{*} C B(Y)_{M}$-module. 
Proof. By facts in the theory of multipliers of an operator space (see e.g. [10, Chapter 4] or [11]), the 'identity map' is a weak* continuous completely contractive homomorphism $\mathcal{M}_{\ell}(Y) \rightarrow C B(Y)$, which maps into $w^{*} C B(Y)_{M}$. If $w^{*} C B(Y)_{M}$ were an operator algebra, and if $Y$ is a left operator $w^{*} C B(Y)_{M}$-module (with the canonical action), then by the aforementioned theory there exist a completely contractive homomorphism $\pi: w^{*} C B(Y)_{M} \rightarrow \mathcal{M}_{\ell}(Y)$ with $\pi(T)(y)=T(y)$ for all $y \in Y, T \in w^{*} C B(Y)_{M}$. That is, $\pi(T)=T$. Thus $w^{*} C B(Y)_{M}=\mathcal{M}_{\ell}(Y)$, and it is clear from the Krein-Smulian theorem and [10, Theorem 4.7.4 (2)] that $w^{*} C B(Y)_{M}$ is weak*-closed in $C B(Y)$.

We now show that $w^{*} C B(Y)_{M}$ is an operator algebra, by appealing to the abstract characterization of operator algebras [10, Theorem 2.3.2]. If $S=\left[S_{i j}\right], T=$ $\left[T_{i j}\right] \in M_{n}\left(w^{*} C B(Y)_{M}\right)$, then one may use the idea in [2, Theorem 2.7] or [12, Theorem 4.9] to write the matrix $a=\left[\sum_{k} S_{i k} T_{k j}\left(y_{p q}\right)\right]$ as an iterated weak* limit of a product of three matrices. The norm of this last product is dominated by $\left\|\left[S_{i j}\right]\right\|\left\|\left[T_{i j}\right]\right\|\left\|\left[y_{p q}\right]\right\|$. It follows by Alaoglu's theorem that $\|a\| \leq\|S\|\|T\|\left\|\left[y_{p q}\right]\right\|$, and thus $\|S T\| \leq\|S\|\|T\|$, as desired.

A similar argument shows that $Y$ is a left operator $w^{*} C B(Y)_{M}$-module: If $T$ is as above, and $y=\left[y_{i j}\right] \in M_{n}(Y)$, then $z=\left[\sum_{k} T_{i k}\left(y_{k j}\right)\right]$ may be written as a weak* limit of a product of two matrices, the latter product having norm $\leq\|T\|\|y\|$. Applying Alaoglu's theorem gives $\|z\| \leq\|T\|\|y\|$, as desired.

The final assertion now follows from [10, Lemma 4.7.5].

Theorem 2.4. Suppose that $Y$ is a right $w^{*}$-rigged module over a dual operator algebra $M$. Suppose that $H$ is a Hilbert space, and that $\theta: M \rightarrow B(H)$ is a normal representation. Then $Y \otimes_{M}^{\sigma h} H^{c}$ is a column Hilbert space. Moreover, the finite rank tensors $Y \otimes H^{c}$ are norm dense in the latter space.

Proof. Let $e_{\alpha}=\phi_{\alpha} \psi_{\alpha}$ (notation as in Definition 2.2). By [8, Lemma 2.5] and Theorem 2.3, $Y \otimes_{M}^{\sigma h} H^{c}$ is a left dual $\mathcal{M}_{\ell}(Y)$-module. By the functoriality of the module normal Haagerup tensor product, we obtain a net of complete contractions $\phi_{\alpha} \otimes I_{H}: Y \otimes_{M}^{\sigma h} H^{c} \rightarrow C_{n(\alpha)}(M) \otimes_{M}^{\sigma h} H^{c}$ and $\psi_{\alpha} \otimes I_{H}: C_{n(\alpha)}(M) \otimes_{M}^{\sigma h} H^{c} \rightarrow Y \otimes_{M}^{\sigma h} H^{c}$. Their composition $\left(\phi_{\alpha} \otimes I_{H}\right)\left(\psi_{\alpha} \otimes I_{H}\right)=e_{\alpha} \otimes I_{H}$ may be regarded as the canonical left action of $e_{\alpha} \in \mathcal{M}_{\ell}(Y)$ on $Y \otimes_{M}^{\sigma h} H^{c}$ mentioned at the start of the proof. Since the action is separately weak* continuous, the composition converges to the identity map on $Y \otimes_{M}^{\sigma h} H^{c}$ in the $w^{*}$-topology (i.e. point-weak* on $Y \otimes_{M}^{\sigma h} H^{c}$ ). However, for any $m \in \mathbb{N}$, we have from facts in [8] that

$$
\begin{aligned}
C_{m}(M) \otimes_{M}^{\sigma h} H^{c} & \cong\left(C_{m} \otimes^{\sigma h} M\right) \otimes_{M}^{\sigma h} H^{c} \\
& \cong C_{m} \otimes^{\sigma h}\left(M \otimes_{M}^{\sigma h} H^{c}\right) \cong C_{m} \otimes^{\sigma h} H^{c} \cong C_{m}\left(H^{c}\right) .
\end{aligned}
$$

However, $C_{m}\left(H^{c}\right)$ is a column Hilbert space. Thus by Lemma 2.1 $Y \otimes_{M}^{\sigma h} H^{c}$ is a column Hilbert space. The last assertion follows from [8, Section 2] and Mazur's theorem that the norm closure of a convex set equals its weak closure.

Henceforth in this section, we stick to the case that $M$ is a $W^{*}$-algebra.

The following is the 'Banach-module' characterization of $W^{*}$-modules promised in our title. The result may be compared with e.g. [10, Corollary 8.5.25]. 
Theorem 2.5. Let $M$ be a $W^{*}$-algebra.

(i) If $Y$ is a dual Banach space and a right $M$-module, then $Y$ is a $W^{*}$-module if and only if there exists a net of integers $(n(\alpha))$, and $w^{*}$-continuous contractive $M$-module maps $\phi_{\alpha}: Y \rightarrow C_{n(\alpha)}(M)$ and $\psi_{\alpha}: C_{n(\alpha)}(M) \rightarrow Y$, with $\psi_{\alpha}\left(\phi_{\alpha}(y)\right) \rightarrow y$ weak ${ }^{*}$ in $Y$, for all $y \in Y$.

(ii) If the conditions in (i) hold, then the weak ${ }^{*}$-limit $w^{*} \lim _{\alpha} \phi_{\alpha}(y)^{*} \phi_{\alpha}(z)$ exists in $M$ for $y, z \in Y$ and equals the $W^{*}$-module inner product.

(iii) An operator $M$-module $Y$ is $w^{*}$-rigged if and only if $Y$ is a $W^{*}$-module, and the matrix norms for $Y$ coincide with the $W^{*}$-module's canonical operator space structure.

Proof. If $Y$ is a $W^{*}$-module, then the existence of the nets in (i) or (iii) follows easily from e.g. Paschke's result [10, Corollary 8.5.25] or [5, Theorem 2.1].

For the other direction in (iii), we follow the proof on pp. 286-287 in [4]. Let $\phi_{\alpha}$ and $\psi_{\alpha}$ be as in Definition 2.2. We write the $k$ th coordinate of $\phi_{\alpha}$ as $x_{k}^{\alpha}$, where $x_{k}^{\alpha}$ is a $w^{*}$-continuous module map from $Y \rightarrow M$, and we write the $k$ th 'entry' of $\psi_{\alpha}$ as $y_{k}^{\alpha} \in Y$. By hypothesis we have $\sum_{k=1}^{n(\alpha)} y_{k}^{\alpha} x_{k}^{\alpha}(y) \stackrel{w *}{\rightarrow} y$ for every $y \in Y$. Let $H$ be a Hilbert space on which $M$ is normally and faithfully represented, let $y, z \in Y$ and $\zeta, \eta \in H$. By Theorem 2.4 and Lemma 2.1, $K=Y \otimes_{M}^{\sigma h} H$ is a column Hilbert space. Define two canonical maps $\Phi: Y \rightarrow B(H, K)$ and $\Psi: w^{*} C B_{M}(Y, M) \rightarrow B(K, H)$, given respectively by $\Phi(y)(\zeta)=y \otimes \zeta$ and $\Psi(f)(y \otimes \zeta)=f(y) \zeta$. Then it is easily checked (or see Subsection 3.1 for this in a more general setting) that $\Phi$ and $\Psi$ are weak* continuous complete isometries.

Let $e_{\alpha}=\sum_{k=1}^{n(\alpha)} \Phi\left(y_{k}^{\alpha}\right) \Psi\left(x_{k}^{\alpha}\right)$. It is easy to check that $e_{\alpha} \Phi(y)=\Phi\left(\psi_{\alpha} \phi_{\alpha} y\right)$; hence $e_{\alpha} \Phi(y) \stackrel{w^{*}}{\rightarrow} \Phi(y)$ for all $y \in Y$. Hence $e_{\alpha}(y \otimes \zeta) \rightarrow y \otimes \zeta$ weak* $^{*}$ in $K$ for all $y \in Y, \zeta \in H$. It follows by the last assertion of Theorem 2.4 that $e_{\alpha} \rightarrow I_{K}$ WOT in $B(K)$. By a similar argument to that of Theorem 4.4 in [8], we can rechoose the net $\left(e_{\alpha}\right)$ such that $e_{\alpha} \rightarrow I$ strongly on $K$. Continuing to follow the proof in [4, one can deduce, by a small modification of the argument there, that the adjoint of any $\Phi(y) \in \Phi(Y)$ is a weak*-limit of terms in $\Psi\left(C B_{M}(Y, M)\right)$. Thus for $z \in Y$, $\Phi(y)^{*} \Phi(z)$ is a weak ${ }^{*}$-limit of terms in $\Psi\left(w^{*} C B_{M}(Y, M)\right) \Phi(Y)$, and hence is in $M$, being a weak ${ }^{*}$-limit of terms in $M$.

Define $\langle y, z\rangle=\Phi(y)^{*} \Phi(z)$ for $y, z \in Y$. As in [4, $Y$ is a $C^{*}$-module over $M$ and the canonical $C^{*}$-module matrix norms coincides with the operator space structure of $Y$, since $\Phi$ is a complete isometry on $Y$. Since $\Phi$ is $w^{*}$-continuous, it follows that the inner product on $Y$ is separately $w^{*}$-continuous. Hence $Y$ is a $W^{*}$-module, by e.g. Lemma 8.5.4 in [10]. This completes the proof of (iii).

The hypothesis in (i) implies, as in the proof of (2.1), that $\|y\|=\sup _{\alpha}\left\|\phi_{\alpha}(y)\right\|$ for each $y \in Y$. Then we may use (2.1) as the definition of an operator space structure on $Y$. This corresponds to an embedding of $Y$ as a submodule of $\bigoplus_{\alpha}^{\infty} C_{n_{\alpha}}(M)$, which is easily seen to be weak* continuous and hence a weak* homeomorphism. Thus $Y$ becomes a dual operator module, and (i) and (ii) then follow from the proof for (iii), as in [4, but replacing limits by weak* limits.

Remark. Shortly after this paper was first distributed, during conversations with Jon Kraus we found a different proof of part of the last result [9]. We also thank Marius Junge for some answers to our question as to whether one could find a third proof using ultrapowers. It seems that such a proof may be more complicated than the others. 
By a weak* approximate identity in a unital dual Banach algebra $M$, we mean a net $\left\{e_{t}\right\}$ in $M$ such that $e_{t} \stackrel{w^{*}}{\rightarrow} 1$. A weak ${ }^{*}$ iterated approximate identity for $M$ is a doubly indexed net $\left\{e_{(\alpha, \beta)}\right\}$ (where $\beta$ and the directed set indexing $\beta$ may possibly depend on $\alpha$ ), such that for each fixed $\alpha$, the weak*-limit $w^{*} \lim _{\beta} e_{(\alpha, \beta)}$ exists, and $w^{*} \lim _{\alpha} w^{*} \lim _{\beta} e_{(\alpha, \beta)}=1$.

Lemma 2.6. A weak* iterated approximate identity for a dual Banach algebra may be reindexed to become a weak* approximate identity.

Proof. This is a variant of the idea of the proof of [2, Lemma 2.1], and we leave it to the reader. The main new feature is that one has to 'build in' finite subsets of $M_{*}$ into the tuples that constitute the elements of the new directed set.

Theorem 2.7. Let $\left\{Y_{i}\right\}$ be a collection of $W^{*}$-modules over a $W^{*}$-algebra $M$, indexed by a directed set. Let $Y$ be a dual Banach space (resp. dual operator space) and a right module over $M$. Suppose that there exist $w^{*}$-continuous contractive (resp. completely contractive) $M$-module maps $\phi_{i}: Y \rightarrow Y_{i}$ and $\psi_{i}: Y_{i} \rightarrow Y$, such that $\psi_{i}\left(\phi_{i}(y)\right) \stackrel{w^{*}}{\rightarrow} y$ in $Y$, for $y \in Y$. Then $Y$ is a $W^{*}$-module (resp. a $W^{*}$ module with its canonical dual operator space structure). For $y, z \in Y$, the limit $w^{*} \lim _{i}\left\langle\phi_{i}(y), \phi_{i}(z)\right\rangle$ exists in $M$ and equals the $W^{*}$-module inner product $\langle y, z\rangle$.

Proof. As in Theorem 2.5, one can focus on the operator space version. For each $i$ choose nets $\phi_{\alpha_{i}}^{i}, \psi_{\alpha_{i}}^{i}$ for $Y_{i}$ as in Definition 2.2. Let $\phi_{i, \alpha_{i}}^{\prime}=\phi_{\alpha_{i}}^{i} \circ \phi_{i}$, and $\psi_{i, \alpha_{i}}^{\prime}$ $=\psi_{i} \circ \psi_{\alpha_{i}}^{i}$. Reindex the net $\left\{\phi_{i, \alpha_{i}}^{\prime}, \psi_{i, \alpha_{i}}^{\prime}\right\}$ by Lemma 2.6, so that the weak* limit of $\psi_{i, \alpha_{i}}^{\prime} \phi_{i, \alpha_{i}}^{\prime}$ in $w^{*} C B(Y)_{M}$ over the new directed set coincides with the iterated weak ${ }^{*}-\operatorname{limit}^{*} w^{*} \lim _{i} w^{*} \lim _{\alpha_{i}} \psi_{i, \alpha_{i}}^{\prime} \phi_{i, \alpha_{i}}^{\prime}$, which equals $I_{Y}$. This gives a new asymptotic factorization of $I_{Y}$ through spaces of the form $C_{n}(M)$ with respect to which $Y$ is $w^{*}$-rigged. Hence by Theorem 2.5, $Y$ is a $W^{*}$-module, with the inner product

$$
\langle y, z\rangle=w^{*} \lim \left\langle\phi_{\alpha_{i}}^{i}\left(\phi_{i}(y)\right), \phi_{\alpha_{i}}^{i}\left(\phi_{i}(z)\right)\right\rangle,
$$

where the limit is taken over the new directed set. Carefully inspecting the directed set used in Lemma 2.6 (a variant of the one used in [2, Lemma 2.1]), it is easy to argue (and is left as an exercise) that the last inner product equals $w^{*} \lim _{i}\left\langle\phi_{i}(y), \phi_{i}(z)\right\rangle$.

Remark. The same proof as the above establishes the analogue of the last result, but for a dual operator module $Y$ over a unital dual operator algebra $M$, taking the $Y_{i}$ to be $w^{*}$-rigged modules over $M$, and the $\phi_{i}, \psi_{i}$ completely contractive (the conclusion being that $Y$ is $w^{*}$-rigged).

Theorem 2.8. If $Y$ is a right $W^{*}$-module over $M$, and if $Z$ is a left (resp. right) dual operator module over $M$, then $Y \otimes_{M}^{\sigma h} Z \cong w^{*} C B_{M}(\bar{Y}, Z)=C B_{M}(\bar{Y}, Z)$ (resp. $Z \otimes_{M}^{\sigma h} \bar{Y} \cong w^{*} C B(Y, Z)_{M}=C B(Y, Z)_{M}$ ) completely isometrically and $w^{*}$-homeomorphically.

Proof. We will use facts and routine techniques from e.g. [15] or [10, 1.2.26, 1.6.3, Section 3.8]. If $T \in B(Y, Z)_{M}$, and if $\left(e_{\alpha}\right)_{\alpha \in I}$ is an orthonormal basis for $Y$ (see [21] or [10,8.5.23]), note that by [15, Theorem 4.2 and the remark after it] we have

$$
T(y)=T\left(\sum_{\alpha} e_{\alpha}\left\langle e_{\alpha}, y\right\rangle\right)=\sum_{\alpha} T\left(e_{\alpha}\right)\left\langle e_{\alpha}, y\right\rangle=a g(y), \quad y \in Y,
$$


where $a$ is the row with $\alpha$ th entry $T\left(e_{\alpha}\right)$, and $g: Y \rightarrow C_{I}(M)$ has $\alpha$ th entry the function $\left\langle e_{\alpha}, \cdot\right\rangle$. Thus $T$ is the composition of 'left multiplication' by $a \in R_{I}(Z)$, and $g$, both of which are weak* continuous (see e.g. the proof of [10, Corollary 8.5.25]). Thus $w^{*} C B_{M}(Y, Z)=C B_{M}(Y, Z)$. We omit the proof that $Z \otimes_{M}^{\sigma h} \bar{Y} \cong$ $w^{*} C B_{M}(Y, Z)$, since we prove a more general result in Theorem 3.5 .

Corollary 2.9. In the situation of the last theorem, the tensor products $\otimes_{M}^{\sigma h}$ occurring there coincide with Magajna's 'extended' module Haagerup tensor product $\bar{\otimes}_{h M}$ used in [5].

It follows that in all of the results in [5], all occurrences of the 'extended' module Haagerup tensor product $\bar{\otimes}_{h M}$ may be replaced by the 'normal module Haagerup tensor product' $\otimes_{M}^{\sigma h}$. This is interesting, since in many of these results this tensor product also coincides with the most important and commonly used tensor product for $W^{*}$-modules, the 'composition' (or 'fusion') tensor product $Y \bar{\otimes}_{\theta} Z$. Thus our results give a new way to treat this famous 'composition tensor product' (see also [13). Both tensor product descriptions have their own advantages: $\bar{\otimes}_{h M}$ allows one to concretely write elements as infinite sums of a nice form, whereas $\otimes_{M}^{\sigma h}$ has many pleasant general properties (see [17, 8]).

Many tensor product relations from [5] transfer to our setting. For example:

Corollary 2.10. Let $Y, Z$ be right $W^{*}$-modules over $M$ and $N$, respectively, and suppose that $\theta: M \rightarrow B(Z)$ is a normal $*$-homomorphism. Then the 'composition tensor product' $Y \bar{\otimes}_{\theta} Z$ equals $Y \otimes_{M}^{\sigma h} Z$. Also, $C B\left(Y \bar{\otimes}_{\theta} Z\right)_{N} \cong Y \otimes_{M}^{\sigma h} C B(Z)_{N} \otimes_{M}^{\sigma h} \bar{Y}$ completely isometrically and weak ${ }^{*}$ homeomorphically.

Proof. The first assertion is discussed above (following from Theorem 2.8 and [5]). For the second, just as in the proof of this result from [5], Theorem 2.8 gives

$$
C B\left(Y \bar{\otimes}_{\theta} Z\right)_{N} \cong\left(Y \bar{\otimes}_{\theta} Z\right) \otimes_{N}^{\sigma h}\left(Y \bar{\otimes}_{\theta} Z\right)^{-} \cong\left(Y \otimes_{M}^{\sigma h} Z\right) \otimes_{N}^{\sigma h}\left(\bar{Z} \otimes_{M}^{\sigma h} \bar{Y}\right),
$$

which equals $Y \otimes_{M}^{\sigma h}\left(Z \otimes_{N}^{\sigma h} \bar{Z}\right) \otimes_{M}^{\sigma h} \bar{Y} \cong Y \otimes_{M}^{\sigma h} B(Z)_{N} \otimes_{M}^{\sigma h} \bar{Y}$ (see [․ 17]).

Similarly, Theorem 2.8 (twice) and associativity of the tensor product give:

Corollary 2.11. Let $M, N$ be $W^{*}$-algebras, let $Y$ be a right $W^{*}$-module over $M$, and let $W$ (resp. $Z$ ) be a dual operator $N$-M-bimodule (resp. dual right operator $N$ module). Then $C B\left(Y, Z \otimes_{N}^{\sigma h} W\right)_{M} \cong Z \otimes_{N}^{\sigma h} C B(Y, W)_{M}$ completely isometrically and weak* homeomorphically.

\section{Some THEORY OF $w^{*}$-RIGGED MOdULES}

3.1. Basic constructs. We begin with some notation and important constructs, which will be used throughout the rest of our paper. For a $w^{*}$-rigged module $Y$ over a dual operator algebra $M$, define $\tilde{Y}$ to be $w^{*} C B(Y, M)_{M}$. Let $\phi_{\alpha}$ and $\psi_{\alpha}$ be as in Definition 2.2. We write the $k$ th coordinate of $\phi_{\alpha}$ as $x_{k}^{\alpha}$, where $x_{k}^{\alpha}$ is a $w^{*}$-continuous module map from $Y \rightarrow M$, and we write the $k$ th 'entry' of $\psi_{\alpha}$ as $y_{k}^{\alpha} \in Y$. By hypothesis we have $\sum_{k=1}^{n(\alpha)} y_{k}^{\alpha} x_{k}^{\alpha}(y) \stackrel{w^{*}}{\rightarrow} y$ for every $y \in Y$.

We sometimes write $\tilde{Y}$ as $X$, and denote by $(\cdot, \cdot)$ the canonical pairing $\tilde{Y} \times Y \rightarrow$ $M$. This is completely contractive, as one may see using the idea in the proof of Theorem 2.3 (the crux of the matter being that for $f \in \tilde{Y}, y \in Y$ we have $(f, y)=w^{*} \lim _{\alpha} \sum_{k=1}^{n(\alpha)} f\left(y_{k}^{\alpha}\right) x_{k}^{\alpha}(y)$, a limit of a product in $\left.M\right)$. Let $H$ be a Hilbert space on which $M$ is normally and faithfully (completely isometrically) represented. 
Then by Lemma 2.4 $K=Y \otimes_{M}^{\sigma h} H$ is a column Hilbert space. Define two canonical maps $\Phi: Y \rightarrow B(H, K)$ and $\Psi: \tilde{Y} \rightarrow B(K, H)$, given respectively by $\Phi(y)(\zeta)=$ $y \otimes \zeta$ and $\Psi(f)(y \otimes \zeta)=f(y) \zeta$. By the argument at the start of [8, Section 4], $\Phi$ is weak* continuous. The canonical map $Y \times H^{c} \rightarrow K$ being completely contractive, a routine argument gives $\Phi$ completely contractive. By the argument on p. 287 in [4, $\Phi$ is a complete isometry: one obtains as in that calculation,

$$
\left\|\left[\phi_{\alpha}\left(y_{i j}\right)\right]\right\| \leq\left\|\left[\Phi\left(y_{i j}\right)\right]\right\|
$$

so that in the limit, by (2.1), $\left\|\left[y_{i j}\right]\right\| \leq\left\|\left[\Phi\left(y_{i j}\right)\right]\right\|$. The canonical weak* continuous complete contraction

$$
\tilde{Y} \otimes^{\sigma h} K^{c} \cong\left(\tilde{Y} \otimes^{\sigma h} Y\right) \otimes_{M}^{\sigma h} H^{c} \rightarrow M \otimes_{M}^{\sigma h} H^{c} \rightarrow H^{c}
$$

corresponds to a separately weak* continuous complete contraction $\tilde{Y} \times K^{c} \rightarrow H^{c}$. The map $\Psi$ is precisely the induced weak* continuous complete contraction $\tilde{Y} \rightarrow$ $B(K, H)$. We leave it as an exercise that $\Psi$ is a complete isometry.

We define the direct sum $M \oplus^{c} Y$ as in [8, Section 4]. Namely, $\theta: M \oplus Y \rightarrow$ $B(H, K \oplus H)$ defined by $\theta((m, y))(\zeta)=\left(m \zeta, y \otimes_{M} \zeta\right)$, for $y \in Y, m \in M, \zeta \in H$, is a one-to-one $M$-module map, which is a weak* continuous complete isometry when restricted to each of $Y$ and $M$. We norm $M \oplus^{c} Y$ by pulling back the operator space structure via $\theta$. Then $M \oplus^{c} Y$ may be identified with the weak ${ }^{*}$-closed right $M$-submodule $\operatorname{Ran}(\theta)$ of $B(H, H \oplus K)$, and hence it is a dual operator $M$-module.

Lemma 3.1. If $Y$ is a right $w^{*}$-rigged module over $M$, then $M \oplus^{c} Y$ is a right $w^{*}$-rigged module over $M$. Also, $\left(M \oplus^{c} Y\right) \otimes_{M}^{\sigma h} H^{c} \cong(H \oplus K)^{c}$ as Hilbert spaces, for $H, K$ as above.

Proof. Define $\phi_{\alpha}^{\prime}: M \oplus^{c} Y \rightarrow C_{n(\alpha)+1}(M)$ and $\psi_{\alpha}^{\prime}: C_{n(\alpha)+1}(M) \rightarrow M \oplus^{c} Y$ to be $I_{M} \oplus \phi_{\alpha}$ and $I_{M} \oplus \psi_{\alpha}$, respectively. We also view $M \subset B(H)$, identify $Y$ and $\Phi(Y)$, and write $n(\alpha)=n$. One may then view $\phi_{\alpha}^{\prime}(m, y)$, for $m \in M, y \in Y$, as the matrix product of the $(n+1) \times 2$ matrix $I_{H} \oplus \Psi_{n, 1}\left(\left[x_{k}^{\alpha}\right]\right)$ (viewed as an operator from $H \oplus K$ to $H^{(n+1)}$ ), and the $2 \times 1$ matrix with entries $m$ and $\Phi(y)$ (viewed as an operator from $H$ to $H \oplus K)$. Thus it is clear that $\phi_{\alpha}^{\prime}$ is completely contractive. Similarly, we view $\psi_{\alpha}^{\prime}\left(m,\left[m_{k}\right]\right)$, for $m \in M,\left[m_{k}\right] \in C_{n}(M)$, as the matrix product of the $2 \times(n+1)$ matrix $I_{H} \oplus \Phi_{1, n}\left(\left[y_{k}^{\alpha}\right]\right)$ (viewed as an operator from $H^{(n+1)}$ to $H \oplus K)$ and the $(n+1) \times 1$ matrix with entries $m$ and $m_{k}$ (viewed as an operator from $H$ to $\left.H^{(n+1)}\right)$. Thus it is clear that $\psi_{\alpha}^{\prime}$ is completely contractive. It is easy to see that $\psi_{\alpha}^{\prime} \phi_{\alpha}^{\prime} \rightarrow I$ weak ${ }^{*}$ on $M \oplus^{c} Y$. So $M \oplus^{c} Y$ is $w^{*}$-rigged.

The last assertion follows just as in [8, Proposition 4.2].

Lemma 3.2. If $Y$ is a right $w^{*}$-rigged module over $M$, then $\tilde{Y}$ is a weak* closed subspace of $C B(Y, M)_{M}$. Indeed $\tilde{Y}$ is a left $w^{*}$-rigged module over $M$, which is also a dual right operator module over $w^{*} C B(Y)$. The canonical map $(\cdot, \cdot): \tilde{Y} \times Y \rightarrow M$ is completely contractive and separately weak* continuous.

Proof. Let $P$ and $Q$ be the canonical projections from $M \oplus^{c} Y$ onto $Y$ and $M$, respectively, and let $i, j$ be the canonical inclusions of $Y$ and $M$ respectively into $M \oplus^{c} Y$. Then $\Theta(T)=j Q T i P$ defines a weak* continuous completely contractive projection on $\mathcal{M}_{\ell}\left(M \oplus^{c} Y\right)=w^{*} C B\left(M \oplus^{c} Y\right)_{M}$; thus its range is weak* closed. However this range is easily seen to be completely isometric to $w^{*} C B(Y, M)_{M}$. Thus the latter becomes a dual operator space, in which, from [10, Theorem 4.7.4(2)], a bounded net converges in the associated weak* topology iff it converges 
point weak*. It follows easily that $\tilde{Y}$ is a weak* closed subspace of $C B(Y, M)_{M}$ (by the Krein-Smulian theorem, or by using the fact that $\mathcal{M}_{\ell}\left(M \oplus^{c} Y\right)$ is weak* closed in $C B\left(M \oplus^{c} Y\right)$ (see Theorem [2.3) ).

Define nets of weak* continuous maps $f \mapsto\left[f\left(y_{k}^{\alpha}\right)\right] \in R_{n(\alpha)}(M)$ and $\left[m_{k}\right] \mapsto$ $\sum_{k} m_{k} x_{k}^{\alpha} \in \tilde{Y}$. Then it is easy to see that with respect to these, $\tilde{Y}$ satisfies the left module variant of Definition 2.2

Since $w^{*} C B\left(M \oplus^{c} Y\right)_{M}$ is a dual operator algebra, it is easy to see that its '12 -corner' $\tilde{Y}$ is a dual right module over its 2-2-corner $w^{*} C B(Y)$. We have already essentially seen the last part.

Corollary 3.3. We have $Y \cong w^{*} C B_{M}(\tilde{Y}, M)$ completely isometrically and weak* homeomorphically, and as right $M$-modules. That is, $\tilde{\tilde{Y}}=Y$. Also a bounded net $y_{t} \rightarrow y$ weak $^{*}$ in $Y$ iff $\left(x, y_{t}\right) \rightarrow(x, y)$ weak ${ }^{*}$ in $M$ for all $x \in \tilde{Y}$.

Proof. This is easy, using the above and the ideas in [2, 12, and routine weak* topology principles.

We say that a map $T: Y \rightarrow Z$ between $w^{*}$-rigged modules over $M$ is adjointable if there exists $S: \tilde{Z} \rightarrow \tilde{Y}$ such that $(w, T y)=(S w, y)$ for all $y \in Y, w \in \tilde{Z}$. The properties of adjointables in the first three paragraphs of p. 389 of [2] hold in our setting too. Moreover by Corollary 3.3 and the definition of $\tilde{Y}$ we have:

Proposition 3.4. A completely bounded module map between $w^{*}$-rigged modules over $M$ is adjointable iff it is weak* continuous.

For any dual right operator $M$-modules $Y, Z$, set $\mathbb{B}(Y, Z)$ (or $\mathbb{B}(Y, Z)_{M}$ ) to be $w^{*} C B(Y, Z)_{M}$ and set $\mathbb{B}(Y)=w^{*} C B(Y)_{M}$. So $\tilde{Y}=\mathbb{B}(Y, M)$. This use of the $\mathbb{B}(\cdot)$ notation may differ from that used in some of the first author's earlier papers.

We also set $N=Y \otimes_{M}^{\sigma h} \tilde{Y}$. Using the canonical completely contractive and separately weak* continuous map $(\cdot, \cdot): \tilde{Y} \times Y \rightarrow M$, one obtains by facts in 8 , Section 2], a weak* continuous completely contractive map

$$
N \otimes^{\sigma h} N \cong Y \otimes_{M}^{\sigma h}\left(\tilde{Y} \otimes^{\sigma h} Y\right) \otimes_{M}^{\sigma h} \tilde{Y} \rightarrow Y \otimes_{M}^{\sigma h} M \otimes_{M}^{\sigma h} \tilde{Y} \cong N .
$$

This endows $N=Y \otimes_{M}^{\sigma h} \tilde{Y}$ with a separately weak* continuous completely contractive product, so that by [10, Theorem 2.7.9], we have that $N$ is a dual operator algebra. We now show that $N$ is unital. As in [12, 2], the elements $v_{\alpha}=\sum_{k=1}^{n(\alpha)} y_{k}^{\alpha} \otimes x_{k}^{\alpha}$ are in $\operatorname{Ball}(N)$, and for any $y \in Y, x \in \tilde{Y}$ we have in the product above the theorem,

$$
v_{\alpha}(y \otimes x)=\psi_{\alpha}\left(\phi_{\alpha}(y)\right) \otimes x \rightarrow y \otimes x
$$

weak* in $N$. If $v_{\alpha_{t}} \rightarrow v$ is a weak* convergent subnet, then by the last centered formula we have $v(y \otimes x)=y \otimes x$, and it follows that $v u=u$ for all $u \in N$. Similarly $u v=u$. We deduce from this that $N$ has an identity of norm 1. Since such an identity is unique, we must have $v_{\alpha} \rightarrow 1_{N}$ weak*

Theorem 3.5. If $Y$ is a right $w^{*}$-rigged module over $M$, and $Z$ is a right dual operator $M$-module, then $\mathbb{B}(Y, Z)$ is weak ${ }^{*}$ closed in $C B(Y, Z)$. Moreover, $\mathbb{B}(Y, Z) \cong$ $Z \otimes_{M}^{\sigma h} \tilde{Y}$ completely isometrically and weak ${ }^{*}$-homeomorphically. In particular, $\mathbb{B}(Y)$ $\cong Y \otimes_{M}^{\sigma h} \tilde{Y}$ as dual operator algebras, equipping the last space with the product mentioned above. 
Proof. As in the second paragraph after Corollary [3.3, by [8, Section 2], we have the canonical weak* continuous complete contractions

$$
\left(Z \otimes_{M}^{\sigma h} \tilde{Y}\right) \otimes_{M}^{\sigma h} Y \cong Z \otimes_{M}^{\sigma h}\left(\tilde{Y} \otimes_{M}^{\sigma h} Y\right) \rightarrow Z \otimes_{M}^{\sigma h} M \cong Z .
$$

This induces a canonical completely contractive $w^{*}$-continuous linear map $\theta: Z \otimes_{M}^{\sigma h}$ $\tilde{Y} \rightarrow C B(Y, Z)_{M}$, which satisfies $\theta(z \otimes x)(y)=z(x, y)$, and which actually maps into $\mathbb{B}(Y, Z)_{M}$.

In the notation above Theorem 3.5, $N=Y \otimes_{M}^{\sigma h} \tilde{Y}$ is a unital dual operator algebra. Set $W=Z \otimes_{M}^{\sigma h} \tilde{Y}$. The canonical weak* continuous maps

$$
W \otimes^{\sigma h}\left(Y \otimes_{M}^{\sigma h} \tilde{Y}\right) \cong Z \otimes_{M}^{\sigma h}\left(\tilde{Y} \otimes^{\sigma h} Y\right) \otimes_{M}^{\sigma h} \tilde{Y} \rightarrow Z \otimes_{M}^{\sigma h} M \otimes_{M}^{\sigma h} \tilde{Y} \cong W
$$

induce a separately weak* continuous complete contraction $m: W \times N \rightarrow W$. Note that $m\left(z \otimes x, 1_{N}\right)=z \otimes x$ for $z \in Z, x \in \tilde{Y}$, since $m\left(z \otimes x, v_{\alpha}\right)=z \otimes x \psi_{\alpha} \phi_{\alpha} \rightarrow z \otimes x$ weak*. Thus $m\left(u, 1_{N}\right)=u$ for any $u \in W$, and so $m\left(u, v_{\alpha}\right) \rightarrow u$ weak* .

Now define $\mu_{\alpha}: C B(Y, Z)_{M} \rightarrow W: T \mapsto \sum_{k=1}^{n(\alpha)} T\left(y_{k}^{\alpha}\right) \otimes x_{k}^{\alpha}$. This is a weak* continuous complete contraction. We have $\mu_{\alpha}(\theta(z \otimes x))=z \otimes x \psi_{\alpha} \phi_{\alpha}=m(z \otimes$ $\left.x, v_{\alpha}\right) \rightarrow z \otimes x$ weak* $^{*}$ for any $z \in Z, x \in \tilde{Y}$. From the equality in the last line, and weak* density, we have for all $u \in W$ that $\mu_{\alpha}(\theta(u))=m\left(u, v_{\alpha}\right)$. The latter, by the fact at the end of the last paragraph, converges to $u$. Since $\left\|\mu_{\alpha}(\theta(u))\right\| \leq\|\theta(u)\|$ it follows from Alaoglu's theorem that $\theta$ is an isometry. Similarly, $\theta$ is a complete isometry. Since it is weak* continuous, $\theta$ has weak* closed range, and is a weak* homeomorphism. Since $\theta\left(\mu_{\alpha}(T)\right) \rightarrow T$ weak ${ }^{*}$ if $T \in \mathbb{B}(Y, Z)$, we have now proved that $\operatorname{Ran}(\theta)=\mathbb{B}(Y, Z)$. Note that in the case when $Z=Y$ we have that $\theta$ is a homomorphism, because it is so on the weak* dense subalgebra $Y \otimes \tilde{Y}$.

Corollary 3.6. If $Y$ is a right $w^{*}$-rigged module over $M, Z$ is a right dual operator $M$-module, and $I, J$ are cardinals/sets, then

(1) $M_{I, J}(\mathbb{B}(Y, Z)) \cong \mathbb{B}\left(C_{J}^{w}(Y), C_{I}^{w}(Z)\right)$;

(2) $\mathbb{B}\left(C_{I}^{w}(M), Z\right) \cong R_{I}^{w}(Z)$;

(3) $\mathbb{B}\left(Y, C_{I}^{w}(M)\right) \cong C_{I}^{w}(\tilde{Y})$.

Proof. (1) follows easily from the theorem as on p. 391 in 2]. One should also use the fact that $\widetilde{C_{J}^{w}(Y)}=R_{I}^{w}(\tilde{Y}) \cong \tilde{Y} \otimes^{\sigma h} R_{I}$, which we leave to the reader. (2) and (3) are immediate from (1).

Remark. During conversations with Jon Kraus we were able to deduce from Theorem 3.5 that if $Y$ is a $w^{*}$-rigged module over $M$ and $Z_{1}, Z_{2}$ are left dual operator $M$-modules, and if $S_{t} \rightarrow S$ weak* in $w^{*} C B_{M}\left(Z_{1}, Z_{2}\right)$, then $I_{Y} \otimes S_{t} \rightarrow I_{Y} \otimes S$ weak* in $C B\left(Y \otimes_{M}^{\sigma h} Z_{1}, Y \otimes_{M}^{\sigma h} Z_{2}\right)$. A similar result holds if $S_{t} \rightarrow S$ weak* in $w^{*} C B(Y)_{M}$. See [9] for details. This allows one to complete the 'easier direction' of the analogue of one of Morita's famous theorems: dual operator algebras are weak* Morita equivalent iff their categories of dual operator modules are appropriately functorially equivalent. The 'difficult direction' appears in [19].

3.2. The weak linking algebra, and its representations. If $Y$ is a $w^{*}$-rigged module over $M$, with $\tilde{Y}$, set

$$
\mathcal{L}^{w}=\left\{\left[\begin{array}{ll}
a & x \\
y & b
\end{array}\right]: a \in M, b \in \mathbb{B}(Y), x \in \tilde{Y}, y \in Y\right\},
$$


with the obvious multiplication. As in [8, Section 4], one may easily adapt the proof of the analogous fact in [12] to see that there is at most one possible sensible dual operator space structure on this linking algebra. Thus the linking algebra with this structure must coincide with $\mathbb{B}\left(M \oplus^{c} Y\right)$. Another description proceeds as follows. Let $H$ be any Hilbert space on which $M$ is normally and completely isometrically represented, and set $K=Y \otimes_{M}^{\sigma h} H^{c}$. We saw at the start of Section 3 the canonical maps $\Phi: Y \rightarrow B(H, K)$ and $\Psi: \tilde{Y} \rightarrow B(K, H)$.

Proposition 3.7. The weak linking algebra $\mathcal{L}^{w}$ of the $w^{*}$-rigged module may be taken to be the subalgebra of $B(H \oplus K)$ with 'four corners' $\Phi(Y), \Psi(\tilde{Y}), M$, and the weak ${ }^{*}$ closure $N$ in $B(K)$ of $\Phi(Y) \Psi(\tilde{Y})$. In particular, $N$ is completely isometrically isomorphic, via a weak* homeomorphism, to $\mathbb{B}(Y)$.

Proof. Clearly $N$ is a weak* closed operator algebra. Also, $N \Phi(Y) \subset \Phi(Y)$, so that by e.g. 4.6.6 in [10] we have a completely contractive homomorphism $\mu: N \rightarrow$ $\mathcal{M}_{\ell}(Y)$. Conversely, since $Y$ is a dual left operator $\mathcal{M}_{\ell}(Y)$-module by Theorem 2.3. so is $K$ by [8, Lemma 2.5]. Thus by the proof of [10, Theorem 4.7.6], there is a normal representation $\theta: \mathcal{M}_{\ell}(Y) \rightarrow B(K)$. If $y \otimes f$ denotes the obvious operator in $w^{*} C B(Y)$, for $y \in Y, f \in \tilde{Y}$, then $\theta(y \otimes f)\left(y^{\prime} \otimes \zeta\right)=y f\left(y^{\prime}\right) \otimes \zeta=\Phi(y) \Psi(f)\left(y^{\prime} \otimes \zeta\right)$ for all $y^{\prime} \in Y, \zeta \in H$. Thus $\theta(y \otimes f)=\Phi(y) \Psi(f) \in N$. However it is easy to see from the fact that $T \psi_{\alpha} \phi_{\alpha} \rightarrow I_{Y}$ weak $^{*}$, that the span of such $y \otimes f$ is weak* dense in $w^{*} C B(Y)_{M}$, and it follows that $\theta$ maps into a weak* dense subset of $N$. Clearly $\mu(\theta(y \otimes f))=y \otimes f$, and so $\mu \circ \theta=I$. Thus $\theta$ is a complete isometry, and the proof is completed by an application of the Krein-Smulian theorem.

3.3. Tensor products of $w^{*}$-rigged modules. If $Y$ is a right $w^{*}$-rigged module over $M$, and if $Z$ is also a right $w^{*}$-rigged module over a dual operator algebra $\mathcal{R}$, and if $Z$ is a left dual operator $M$-module, then $Y \otimes_{M}^{\sigma h} Z$ is a right dual operator $\mathcal{R}$-module (see [8, Section 2]). As in the proof of Theorem 2.4, we obtain a net of completely contractive right $\mathcal{R}$-module maps $\phi_{\alpha} \otimes I_{Z}: Y \otimes_{M}^{\sigma h} Z \rightarrow C_{n(\alpha)}(M) \otimes_{M}^{\sigma h}$ $Z \cong C_{n(\alpha)}(Z)$, and $\psi_{\alpha} \otimes I_{Z}: C_{n(\alpha)}(Z) \rightarrow Y \otimes_{M}^{\sigma h} Z$, such that the composition $\left(\phi_{\alpha} \otimes I_{Z}\right)\left(\psi_{\alpha} \otimes I_{Z}\right)=e_{\alpha} \otimes I_{Z}$ converges weak* to the identity map on $Y \otimes_{M}^{\sigma h} Z$. By the remark after Theorem 2.7, $Y \otimes_{M}^{\sigma h} Z$ is a $w^{*}$-rigged module over $\mathcal{R}$. In particular, if $\mathcal{R}$ is a $W^{*}$-algebra, then $Y \otimes_{M}^{\sigma h} Z$ is a $W^{*}$-module over $\mathcal{R}$ by Theorem 2.5.

In the setting of the last paragraph (and $\mathcal{R}$ possibly nonselfadjoint again),

$$
\left(Y \otimes_{M}^{\sigma h} Z\right)^{\tilde{2}}=w^{*} C B\left(Y \otimes_{M}^{\sigma h} Z, \mathcal{R}\right)_{\mathcal{R}} \cong \tilde{Z} \otimes_{M}^{\sigma h} \tilde{Y}
$$

completely isometrically and weak* homeomorphically. We give one proof of this (another route is to use the method on pp. 402-403 in [2]). Note that the canonical weak* continuous complete contractions

$$
\left(\tilde{Z} \otimes_{M}^{\sigma h} \tilde{Y}\right) \otimes^{\sigma h}\left(Y \otimes_{M}^{\sigma h} Z\right) \rightarrow \tilde{Z} \otimes_{M}^{\sigma h} M \otimes_{M}^{\sigma h} Z \rightarrow \tilde{Z} \otimes_{M}^{\sigma h} Z \rightarrow \mathcal{R}
$$

induce a weak* continuous complete contraction $\sigma: \tilde{Z} \otimes_{M}^{\sigma h} \tilde{Y} \rightarrow w^{*} C B\left(Y \otimes_{M}^{\sigma h}\right.$ $Z, \mathcal{R})_{\mathcal{R}}$. On the other hand, the complete contraction from the operator space projective tensor product to $Y \otimes_{M}^{\sigma h} Z$ induces a complete contraction $w^{*} C B\left(Y \otimes_{M}^{\sigma h}\right.$ $Z, \mathcal{R})_{\mathcal{R}} \rightarrow C B(Y, C B(Z, \mathcal{R}))$ that is easily seen to map into $C B(Y, \tilde{Z})$, and in fact into $w^{*} C B(Y, \tilde{Z})_{M}$. Now it is easy to check that this map $w^{*} C B\left(Y \otimes_{M}^{\sigma h} Z, \mathcal{R}\right)_{\mathcal{R}} \rightarrow$ $w^{*} C B(Y, \tilde{Z})_{M}$ is also weak* continuous. By Theorem 3.5, we have constructed a weak* continuous complete contraction $\rho: w^{*} C B\left(Y \otimes_{M}^{\sigma h} Z, \mathcal{R}\right)_{\mathcal{R}} \rightarrow \tilde{Z} \otimes_{M}^{\sigma h} \tilde{Y}$. 
It is easy to check that $\rho \sigma=I d$; thus $\sigma$ is completely isometric and by KreinSmulian has weak* closed range. Any $f \in w^{*} C B\left(Y \otimes_{M}^{\sigma h} Z, \mathcal{R}\right)_{\mathcal{R}}$ is a weak ${ }^{*}$ limit of $f \circ\left(\psi_{\alpha} \phi_{\alpha} \otimes I_{Z}\right)$. The latter function is easily checked to lie in $\operatorname{Ran}(\sigma)$, using the fact that for any $y \in Y$ the map $f(y \otimes \cdot)$ on $Z$ is in $\tilde{Z}$. Hence $\sigma$ has weak* dense range, and hence is surjective, proving (3.1).

Just as in the proof of Corollary 2.10, one may deduce from (3.1) the relation $\mathbb{B}\left(Y \otimes_{M}^{\sigma h} Z\right) \cong Y \otimes_{M}^{\sigma h} \mathbb{B}(Z)_{\mathcal{R}} \otimes_{M}^{\sigma h} \tilde{Y}$

In fact the weak* variants of all the theorems in Section 6 of 2 are valid (and we will perhaps present these elsewhere). In the next subsection, we merely focus on 6.8 from that paper, which we shall need at the end of the next section.

3.4. The $W^{*}$-dilation. This important tool is a canonical ' $W^{*}$-module 'envelope' of a $w^{*}$-rigged module $Y$ over $M$. If $\mathcal{R}$ is a $W^{*}$-algebra containing $M$ as a weak*closed subalgebra with $1_{\mathcal{R}}=1_{M}$, then $E=Y \otimes_{M}^{\sigma h} \mathcal{R}$ is a $W^{*}$-module over $\mathcal{R}$ by 3.3. and it is called a $W^{*}$-dilation of $Y$. We may identify $Y$ with $Y \otimes 1$. This is a completely isometric weak* homeomorphic identification, since by (2.1) we have for $\left[y_{i j}\right] \in M_{n}(Y)$ that

$$
\left\|\left[y_{i j} \otimes 1\right]\right\|_{M_{n}(E)}=\sup _{\alpha}\left\|\left[\left(\phi_{\alpha} \otimes I_{Z}\right)\left(y_{i j} \otimes 1\right)\right]\right\|=\sup _{\alpha}\left\|\left[\phi_{\alpha}\left(y_{i j}\right)\right]\right\|=\left\|\left[y_{i j}\right]\right\|_{M_{n}(Y)} .
$$

Thus every $w^{*}$-rigged module over $M$ is a weak* closed $M$-submodule of a $W^{*}$ module over $\mathcal{R}$. Usually we assume that $\mathcal{R}$ is generated as a $W^{*}$-algebra by $M$.

Similarly, it is easy to see that $\tilde{Y}$ is a weak* closed left $M$-submodule of $\mathcal{R} \otimes_{M}^{\sigma h} \tilde{Y}$. By (3.1) above, $\mathcal{R} \otimes_{M}^{\sigma h} \tilde{Y}=\tilde{E}$, which in turn is just the conjugate $C^{*}$-module (see e.g. [10, 8.1.1 and 8.2.3(2)]) $\bar{E}$ of $E$. We claim that $\mathbb{B}(Y)$ may be regarded as a weak* closed subalgebra of $\mathbb{B}(E)$ having a common identity element. By a principle we have met several times now (e.g. in the proof of Proposition 3.7), there is a canonical weak* continuous completely contractive unital homomorphism $\mathbb{B}(Y) \rightarrow \mathbb{B}(E)$. However, since $Y \cong Y \otimes 1 \subset E$ as above, it is easy to see that the last homomorphism is a completely isometric weak* homeomorphism. Thus we have established the variant in our setting of [2, Theorem 6.8].

The $W^{*}$-dilation is studied in a more general setting in [19, 18].

3.5. Direct sums. If $Y$ is a $w^{*}$-rigged module over $M$, and if $P \in \mathbb{B}(Y)$ is a contractive idempotent, then it is easy to see from the Remark after Theorem 2.7. that $P(Y)$ is a $w^{*}$-rigged module over $M$, called an orthogonally complemented submodule of $Y$.

As in the discussion at the top of p. 409 of [2], if $\left\{Y_{k}\right\}_{k \in I}$ is a collection of $w^{*}$-rigged modules over $M$, and if $E_{k}=Y_{k} \otimes_{M}^{\sigma h} \mathcal{R}$ is the $W^{*}$-dilation of $Y_{k}$, for a $W^{*}$-algebra $\mathcal{R}$ containing $M$, then we can define the 'column direct sum' $\bigoplus_{k \in I}^{c} Y_{k}$, to be $\bigoplus_{k \in I}^{c} Y_{k}=\left\{\left(y_{k}\right) \in \bigoplus_{k \in I}^{c} E_{k}: y_{k} \in Y_{k}\right.$ for all $\left.k \in I\right\}$, where $\bigoplus_{k \in I}^{c} E_{k}$ is the $W^{*}$-module direct sum (see [21] or [10, 8.5.26]). A key principle, which is used all the time when working with this direct sum, is the following. Consider the directed set of finite subsets $\Delta$ of $I$, and for $z \in \bigoplus_{k \in I}^{c} E_{k}$, write $z_{\Delta}$ for the tuple $z$, but with entries $z_{k}$ switched to zero if $k \notin \Delta$. Then $\left(z_{\Delta}\right)_{\Delta}$ is a net indexed by $\Delta$, which converges weak $*$ to $z$. For example, it follows from this principle that $\bigoplus_{k \in I}^{c} Y_{k}$ is the weak* closure inside $\bigoplus_{k \in I}^{c} E_{k}$ of the 'finitely supported tuples' $\left(y_{k}\right)$ with $y_{k} \in Y_{k}$ for all $k$. 
Theorem 3.8. If $\left\{Y_{k}: k \in I\right\}$ is a collection of $w^{*}$-rigged modules over $M$, then $\bigoplus_{k \in I}^{c} Y_{k}$ is again a $w^{*}$-rigged module over $M$. Also $\widetilde{\bigoplus_{k \in I}^{c} Y_{k}}=\bigoplus_{k \in I}^{r} \tilde{Y}_{k}$.

Proof. We first observe that this is easy if $I$ is finite. The reader may just want to consider the case of two modules, which is similar to the proof of Lemma 3.1. In fact one may use $\$ 4.5$ below to see quickly that $Y_{1} \oplus^{c} Y_{2}$ is $w^{*}$-rigged if $Y_{1}$ and $Y_{2}$ are: note that if $E=E_{1} \bigoplus^{c} E_{2}$ in the notation of the last paragraph, and if $\left(z_{1}, z_{2}\right) \in E$ is such that $\left\langle\left(z_{1}, z_{2}\right) \mid\left(y_{1}, y_{2}\right)\right\rangle=\left\langle z_{1} \mid y_{1}\right\rangle+\left\langle z_{2} \mid y_{2}\right\rangle \in M$ for all $y_{1}, y_{2} \in Y$, then $z_{k}^{*} \in X_{k}=\tilde{Y}_{k}$ by 4.5 . It is then easy to see that the conditions of 4.5 are satisfied, so that $Y_{1} \bigoplus^{c} Y_{2}$ is $w^{*}$-rigged.

Suppose that $I$ is infinite. In the notation of the paragraph above the theorem, we have that $\bigoplus_{k \in \Delta}^{c} Y_{k}$ is $w^{*}$-rigged by the last paragraph. There are canonical maps $\phi_{\Delta}$ and $\psi_{\Delta}$ between $Y=\bigoplus_{k \in I}^{c} Y_{k}$ and $\bigoplus_{k \in \Delta}^{c} Y_{k}$. Namely, $\phi_{\Delta}$ is essentially the map $z \mapsto z_{\Delta}$ and $\psi_{\Delta}$ is the 'inclusion'; indeed $\phi_{\Delta} \circ \psi_{\Delta}=I d$. It is easy to see that these maps are completely contractive and weak* continuous, since when one tensors them with $I_{\mathcal{R}}$ they have these properties. Also, $\psi_{\Delta} \circ \phi_{\Delta} \rightarrow I_{Y}$ weak $^{*}$, using the principle above the theorem that $z_{\Delta} \rightarrow z$. It follows from the Remark after Theorem 2.7 that $Y$ is $w^{*}$-rigged. We leave the last relation to the reader.

The following universal property shows that the direct sum $\bigoplus_{k \in I}^{c} Y_{k}$ is not dependent on the specific construction of it above:

Theorem 3.9. Suppose that $\left\{Y_{k}\right\}_{k \in I}$ is a collection of dual operator modules over $M$, that $Y$ is a fixed $w^{*}$-rigged module over $M$, and that there exist weak ${ }^{*}$ continuous completely contractive $M$-module maps $i_{k}: Y_{k} \rightarrow Y, \pi_{k}: Y \rightarrow Y_{k}$ with $\pi_{k} \circ i_{m}=$ $\delta_{k m} I d_{Y_{m}}$ for all $k, m$. Here $\delta_{k m}$ is the Kronecker delta. Then each $Y_{k}$ is $w^{*}$-rigged, and $Y$ is completely isometrically weak ${ }^{*}$ homeomorphically, $M$-isomorphic to the column direct sum $Z \oplus^{c}\left(\bigoplus_{k}^{c} Y_{k}\right)$ defined above, where $Z$ is a submodule of $Y$ which is also $w^{*}$-rigged. If $\sum_{k \in I} i_{k} \pi_{k}=I_{Y}$ in the weak ${ }^{*}$-topology of $\mathbb{B}(Y)$, then $Z=(0)$.

Proof. The ranges $i_{k}\left(Y_{k}\right)$ are orthogonally complemented submodules of $Y$, and hence they are $w^{*}$-rigged, and so is $Y_{k}$. The partial sums of $R=\sum_{k} i_{k} \pi_{k}$ form an increasing net of contractive projections in the dual operator algebra $\mathbb{B}(Y)$, indexed by the finite subsets of $I$ directed upwards by inclusion. Hence it converges in the weak* topology in $\mathbb{B}(Y)$ to a contractive projection $R \in \mathbb{B}(Y)$. Let $Z=$ $\operatorname{Ran}(I-R)$, which again is $w^{*}$-rigged. Define $Z \oplus^{c}\left(\bigoplus_{k}^{c} Y_{k}\right)$ as above the theorem to be a weak* closed $M$-submodule of the $W^{*}$-module direct sum $\left(Z \otimes_{M}^{\sigma h} \mathcal{R}\right) \oplus^{c}$ $\left(\bigoplus_{k}^{c}\left(Y_{k} \otimes_{M}^{\sigma h} \mathcal{R}\right)\right)$. Tensoring all maps with $I_{\mathcal{R}}$, we obtain maps back and forth between $Y_{k} \otimes_{M}^{\sigma h} \mathcal{R}$ and $Y \otimes_{M}^{\sigma h} \mathcal{R}$, and between $Z \otimes_{M}^{\sigma h} \mathcal{R}$ and $Y \otimes_{M}^{\sigma h} \mathcal{R}$, satisfying the hypotheses of [5, Theorem 2.2]. Note that $i_{k} \pi_{k} \in \mathbb{B}(Y)_{M}$, and $Y \otimes_{M}^{\sigma h} \mathcal{R}$ is a left dual operator $\mathbb{B}(Y)_{M}$-module (since $Y$ is). It follows that $\sum_{k}\left(i_{k} \pi_{k} \otimes I_{\mathcal{R}}\right)=R \otimes I_{\mathcal{R}}$, so that $\sum_{k}\left(i_{k} \pi_{k} \otimes I_{\mathcal{R}}\right)+(I-R) \otimes I_{\mathcal{R}}=I$. From [5, Theorem 2.2], it follows that the canonical map is a completely isometric weak* homeomorphic, $\mathcal{R}$-isomorphism between $\left(Z \otimes_{M}^{\sigma h} \mathcal{R}\right) \oplus^{c}\left(\bigoplus_{k}^{c}\left(Y_{k} \otimes_{M}^{\sigma h} \mathcal{R}\right)\right)$ and $Y \otimes_{M}^{\sigma h} \mathcal{R}$. Its restriction to the copy of $Z \oplus\left(\oplus_{n}^{w} Y_{n}\right)$ is the desired map.

As in [2, Section 7] it follows that the column direct sum is associative and commutative. We also have the obvious variant of [2, Theorem 7.4] valid in our setting, concerning the direct sum $\bigoplus_{k} T_{k}$ of maps $T_{k} \in \mathbb{B}\left(Y_{k}, Z_{k}\right)$. Again, the proof of this is now familiar: apply the $W^{*}$-module case of this result to the maps $T_{k} \otimes I_{\mathcal{R}}$ between the $W^{*}$-dilations, and then restrict to the appropriate subspace. 
Also, we obtain from Theorem 3.9 and functoriality of the tensor product $\otimes_{M}^{\sigma h}$, as in [2, p. 411], both left and right distributivity of this tensor product $\otimes_{M}^{\sigma h}$ over column direct sums of $w^{*}$-rigged modules:

$$
\left(\bigoplus_{k}^{c} Y_{k}\right) \otimes_{M}^{\sigma h} Z \cong \bigoplus_{k}^{c}\left(Y_{k} \otimes_{M}^{\sigma h} Z\right)
$$

and

$$
Y \otimes_{M}^{\sigma h}\left(\bigoplus_{k}^{c} Z_{k}\right) \cong \bigoplus_{k}^{c}\left(Y \otimes_{M}^{\sigma h} Z_{k}\right) .
$$

All spaces in these formulae are right $w^{*}$-rigged modules, and $Z$ and $Z_{k}$ are also left dual operator $M$-modules. For the last formula, one may use the fact that if $T_{t} \rightarrow T$ weak* in $\mathbb{B}(Z)$, then $I_{Y} \otimes T_{t} \rightarrow I \otimes T$ weak* in $\mathbb{B}\left(Y \otimes_{M}^{\sigma h} Z\right)$. Indeed, if we have a weak* convergent subnet $I_{Y} \otimes T_{t_{\mu}} \rightarrow S \in \mathbb{B}\left(Y \otimes_{M}^{\sigma h} Z\right)$, then $S(y \otimes z)=y \otimes T(z)$ for $y \in Y, z \in Z$. Since finite rank tensors are weak* dense, we have $S=I \otimes T$, and it follows that $I_{Y} \otimes T_{t} \rightarrow I \otimes T$ weak*. Full details are left to the reader.

Remark. Theorem 3.9 also shows that the definition from Section 3 of $M \oplus^{c} Y$ agrees with the column direct sum in the present subsection. Thus the last relation in Lemma 3.1 is a simple special case of the second (last) centered formula above.

\section{Equivalent Definitions of $w^{*}$-RigGed MOdules}

4.1. The reader may prefer some of the following four descriptions of $w^{*}$-rigged modules, each of which involves a pair $X, Y$ of modules. In each case, the first paragraph of the subsection constitutes the alternative definition. In the second and following paragraphs the equivalence with the original definition is sketched. One must show that every $w^{*}$-rigged module $Y$ satisfies (or is completely isometrically, weak* homeomorphically, $M$-isomorphic to a module which satisfies) the given alternative description, and that conversely any $Y$ satisfying the description is $w^{*}$ rigged, and that moreover $X \cong \tilde{Y}$.

We will be a little informal in this section, since the objectives here are quite clear: we are just adapting four theorems from [2, Section 5] to the weak* topology setting of the present paper. The reader will easily be able to add any missing details.

4.2. Second definition of a $w^{*}$-rigged module. Fix two unital dual operator algebras $M$ and $N$, and two dual operator bimodules $X$ and $Y$, with $X$ an $M-N$ bimodule and $Y$ an $N$-M-bimodule. We also assume that there exists a separately weak*-continuous completely contractive $M$-bimodule map $(\cdot, \cdot): X \times Y \rightarrow M$, which is balanced over $N$, and a separately weak*-continuous completely contractive $N$-bimodule map $[\cdot, \cdot]: Y \times X \rightarrow N$, which is balanced over $M$, such that $(x, y) x^{\prime}=$ $x\left[y, x^{\prime}\right]$ and $y^{\prime}(x, y)=\left[y^{\prime}, x\right] y$ for $x, x^{\prime} \in X, y, y^{\prime} \in Y$, and such that $[\cdot, \cdot]$ induces a weak* continuous quotient map $Y \otimes^{\sigma h} X \rightarrow N$.

As in [2, Section 5], any $w^{*}$-rigged module in the sense of Definition 2.2 satisfies the conditions in the last paragraph, with $N=\mathbb{B}(Y)$ (or $N=Y \otimes_{M}^{\sigma h} \tilde{Y}$ ), and $X=$ $\tilde{Y}$, by our earlier results. Conversely, given the conditions in the last paragraph, suppose that $u \in \operatorname{Ball}\left(Y \otimes^{\sigma h} X\right)$ maps to $1_{N}$, and that $\left(f_{s}\right)$ is a net of finite rank tensors in $\operatorname{Ball}\left(Y \otimes_{\mathrm{h}} X\right)$ which converges weak* to $u$ (using [8, Corollary 2.6]). The image of $f_{s}$ in $N$ converges weak ${ }^{*}$ to $1_{N}$. From this we see that $Y$ satisfies Definition 2.2 (as in similar assertions in 2] (see e.g. bottom of p. 384 there)). Moreover, by 
a now routine modification of the last two paragraphs of the proof of 12 , Theorem 4.1], one sees that the canonical map $X \rightarrow w^{*} C B(Y, M)_{M}$ is a weak* continuous surjective complete isometry. That is, $X \cong \tilde{Y}$ as dual operator $M$-modules. We have a canonical weak* continuous complete quotient map $\theta: Y \otimes_{M}^{\sigma h} \tilde{Y} \rightarrow N$. A simple modification of the last paragraph of the proof of Theorem 3.5. which is essentially the proof of $(\Leftarrow)$ in $[8$, Theorem 3.3], shows that $\theta$ is a complete isometry, so that $[\cdot, \cdot]$ induces a weak* homeomorphic complete isometry $Y \otimes_{M}^{\sigma h} \tilde{Y} \cong N$.

4.3. Third definition of a $w^{*}$-rigged module. A pair consisting of a dual left $M$-module $X$, and a dual right $M$-module $Y$, with a separately weak* continuous completely contractive pairing $(\cdot, \cdot): X \times Y \rightarrow M$, such that if we equip $N=$ $Y \otimes_{M}^{\sigma h} X$ with the canonical separately weak* continuous completely contractive product induced by $(\cdot, \cdot)$, as in the discussion above Theorem 3.5, then this (dual operator) algebra has an identity of norm 1 . We also assume that the canonical actions of $N$ on $Y$ and on $X$ are nondegenerate (that is, $1_{N} y=y, x 1_{N}=x$ for $y \in Y, x \in X)$.

Again, clearly any $w^{*}$-rigged module in the earlier sense satisfies the conditions in the last paragraph, by Theorem 3.5 and the remarks above it. Conversely, suppose that $X, Y,(\cdot, \cdot)$ are as in the last paragraph; we shall verify the conditions of Definition 4.2. It is by-now-routine to see that $X, Y$ are dual operator modules over $N$. To see that $(\cdot, \cdot)$ is $N$-balanced, one shows that for $x \in X, y \in Y$, the two weak ${ }^{*}$ continuous functions $(x, \cdot y)$ and $(x \cdot y)$ on $N$, are equal on the weak ${ }^{*}$ dense subset $Y \otimes X$ of $N$. The rest is obvious.

4.4. Fourth description of $w^{*}$-rigged modules. Let $M, N$ be weak ${ }^{*}$ closed unital subalgebras of $B(H)$ and $B(K)$, respectively, for Hilbert spaces $H, K$, and let $X \subset B(K, H), Y \subset B(H, K)$ be weak* closed subspaces, such that the associated subset $\mathcal{L}$ of $B(H \oplus K)$ is a subalgebra of $B(H \oplus K)$, for Hilbert spaces $H, K$. This is the same as specifying a list of obvious algebraic conditions, such as $X Y \subset M$. Assume in addition that the weak ${ }^{*}$ closure $N$ in $B(K)$ of $Y X$, possesses a net $\left(e_{t}\right)$ with terms of the form $y x$, for $x \in \operatorname{Ball}\left(C_{n}(X)\right)$ and $y \in \operatorname{Ball}\left(R_{n}(Y)\right)$, such that $e_{t} \rightarrow 1_{N}$ weak*.

That every $w^{*}$-rigged module $Y$ is essentially of this form follows by replacing $Y$ and $\tilde{Y}$ by $\Phi(Y)$ and $X=\Psi(\tilde{Y})$, respectively, and looking at the weak linking algebra $\mathcal{L}^{w}$ at the end of Section 3.2. Let $e_{\alpha}=\sum_{k=1}^{n(\alpha)} \Phi\left(y_{k}^{\alpha}\right) \Psi\left(x_{k}^{\alpha}\right)$. It is easy to check that $e_{\alpha} \Phi(y)=\Phi\left(\psi_{\alpha} \phi_{\alpha} y\right)$; hence $e_{\alpha} \Phi(y) \stackrel{w^{*}}{\rightarrow} \Phi(y)$ for all $y \in Y$. Hence $e_{\alpha}(y \otimes \zeta) \rightarrow y \otimes \zeta$ weak $^{*}$ in $K$ for all $y \in Y, \zeta \in H$. It follows by the last assertion of Theorem 2.4 that $e_{\alpha} \rightarrow I_{K}$ WOT in $B(K)$. This gives the condition in the last paragraph. Conversely, given the setup in the last paragraph, we will verify the conditions of $\$ 4.2$ The canonical map $\theta: Y \otimes^{\sigma h} X \rightarrow N$ is completely contractive and weak* continuous, we need to show it is a quotient map. If $T \in \operatorname{Ball}(N)$, and if we write the $x$ and $y$ in the last paragraph as $x=\left[x_{k}\right], y=\left[y_{k}\right]$, then $u_{t}=\sum_{k} T y_{k} \otimes x_{k} \in$ $\operatorname{Ball}\left(Y \otimes^{\sigma h} X\right)$. Consider a weak* convergent subnet $u_{t_{\beta}} \rightarrow u \in \operatorname{Ball}\left(Y \otimes^{\sigma h} X\right)$. Then $\theta\left(u_{t_{\beta}}\right) \rightarrow \theta(u)$. On the other hand, $\theta\left(u_{t_{\beta}}\right)=T e_{t_{\beta}} \rightarrow T$ weak $^{*}$. So $T=\theta(u)$, so that $\theta$ is a quotient map.

4.5. Fifth definition of a $w^{*}$-rigged module. Let $\mathcal{R}$ be a $W^{*}$-algebra containing $M$ as a weak* closed subalgebra with $1_{\mathcal{R}}=1_{M}$, and suppose that $Z$ is a right $W^{*}$-module over $\mathcal{R}$, and that $Y$ is a weak* closed $M$-submodule of $Z$. Define 
$W=\{z \in Z:\langle z \mid y\rangle \in M\}$, and set $N$ to be the weak* closure in $\mathbb{B}(Z)_{\mathcal{R}}$ of the span of terms of the form $|y\rangle\langle w|$ for $y \in Y, w \in W$ (here $|y\rangle\langle w|$ is the obvious 'rank one' operator $z \mapsto y\langle w, z\rangle$ on $Z$. Suppose that there is a net $\left(e_{t}\right)$ converging to $I_{Z}$ weak* in $\mathbb{B}(Z)$, with terms of the form $e_{t}=\sum_{k=1}^{n}\left|y_{k}\right\rangle\left\langle w_{k}\right|$, where $y_{k} \in Y, w_{k} \in W$ with $\sum_{k=1}^{n}\left|y_{k}\right\rangle\left\langle y_{k}\right| \leq 1$ and $\sum_{k=1}^{n}\left|w_{k}\right\rangle\left\langle w_{k}\right| \leq 1$.

We claim that under the hypotheses in the last paragraph, $Y$ is $w^{*}$-rigged, $\tilde{Y}=$ $\{\bar{w} \in \bar{Z}: w \in W\}$, and $\mathbb{B}(Y)_{M} \cong N$. To see this, we follow the proof of [2, Theorem 5.10], working inside the linking $W^{*}$-algebra $\mathcal{L}^{w}(Z)$ for $Z$, where all inner products and module actions become concrete operator multiplication. Note first that $W$ is a weak* closed right $M^{*}$-submodule of $Z$, and hence $X=W^{*}$ is a weak ${ }^{*}$ closed left $M$-submodule of $Z^{*}$. The subspace of $\mathcal{L}^{w}(Z)$ with four corners $M, X, Y, N$, is a weak* closed subalgebra, and it is easy to see that the criteria of $\$ 4.4$ are met, for these subspaces of $\mathcal{L}^{w}(Z)$. Hence the criteria of $\$ 4.2$ are met, and we are done by facts from that place.

Conversely, to see that every $w^{*}$-rigged module $Y$ is essentially of this form, set $Z=Y \otimes_{M}^{\sigma h} \mathcal{R}$, which we saw in Proposition 3.4 is a $W^{*}$-module over $\mathcal{R}$, containing $Y$ as a weak* closed $M$-submodule. Also we saw that $\tilde{Y} \subset \bar{Z}$ (resp. $\left.\mathbb{B}(Y)_{M} \subset \mathbb{B}(Z)_{\mathcal{R}}\right)$ as a weak* closed $M$-submodule (resp. weak* closed subalgebra with common identity). Now apply a simple variant of the argument in the last paragraph of [2, p. 405].

\section{EXAmples}

(1) As we saw in Section $2, W^{*}$-modules are $w^{*}$-rigged. Thus so are WTROs, where a WTRO is a weak* closed space $Z$ of Hilbert space operators with $Z Z^{*} Z \subset Z$ (see [10, 8.5.11 and 8.5.18]).

(2) For finite dimensional modules over a finite dimensional operator algebra $M$, the notions of 'rigged' and ' $w^{*}$-rigged' coincide, as is easily seen from Definition 2.2,

(3) By e.g. 4.2 and [8, Theorem 3.3], every weak* Morita equivalence bimodule in the sense of $[8]$ is $w^{*}$-rigged. In Section 3 of that paper, a long list of examples of these bimodules is given. Indeed a weak* Morita equivalence bimodule is essentially the same thing as a 'left-right symmetric' variant of 4.2 (that is, we also assume there that $(\cdot, \cdot)$ induces a weak* continuous quotient map $X \otimes^{\sigma h} Y \rightarrow M$ ).

There are simple examples of $w^{*}$-rigged modules which give rise to no kind of weak* Morita equivalence (in contrast to the $W^{*}$-module case). For example, consider $Y=R_{2}$, a right $w^{*}$-rigged module over the upper triangular $2 \times 2$ matrices. A partial result in the positive direction here: if $Y$ is a $w^{*}$-rigged $M$-module which is $w^{*}-f u l l$, that is, the span of the range of $(\cdot, \cdot)$ is weak* dense in $M$, and if $\mathcal{R}$ is a $W^{*}$-algebra generated by $M$, then the $W^{*}$-dilation $E=Y \otimes_{M}^{\sigma h} \mathcal{R}$ gives a von Neumann algebraic Morita equivalence (see [22] or [10, Section 8.7]) between $\mathcal{R}$ and $\mathbb{B}(E)$. This will follow if $E$ is $w^{*}$-full over $\mathcal{R}$ (see [10, 8.5.12]). To this end, note that $\bar{E}=\tilde{E}=\mathcal{R} \otimes_{M}^{\sigma h} \tilde{Y}$ by (3.1). Thus $\tilde{Y} Y$, and therefore also $M$, is contained in the weak* closure of $\tilde{E} E$. So $E$ is $w^{*}$-full, since the latter is an ideal of $\mathcal{R}$, and because $M$ generates $\mathcal{R}$.

(4) The second dual of a rigged module over an operator algebra $A$ is $w^{*}$-rigged over $A^{* *}$. This is evident by taking the second dual of all objects in the 
definition of a rigged module from [6] say (note that $C_{n}(A)^{* *}=C_{n}\left(A^{* *}\right)$ by basic operator space duality).

(5) If $P$ is a weak*-continuous completely contractive idempotent $M$-module map on $C_{I}^{w}(M)$, for a cardinal/set $I$, then $\operatorname{Ran}(P)$ is a $w^{*}$-rigged module (see the first paragraph of Section 3.5).

(6) Examples of $w^{*}$-rigged may be built analogously to the rigged modules constructed in [7] (see e.g. the end of Section 6 there).

(7) In [8] (see also [18]) we defined a stronger variant of weak* Morita equivalence (see Example (3) above) called weak Morita equivalence. In just the same way, we can define a subclass of $w^{*}$-rigged modules: we say that an $M$-module is weakly rigged if it satisfies Definition 3.2 of [8], but with the phrase '(strong) Morita context' replaced by '(P)-context' (see [12, p. 20]). An adaption of the proof of [8, Corollary 3.4] shows that weakly rigged modules are $w^{*}$-rigged. One may then show that any weakly rigged module pair $(Y, X)$ arises as a weak* closure of a rigged module situation, just as in Example (2) after Definition 3.2 of [ $[8$, but dropping the requirement on the cai for $A$ there. This proceeds by showing that the linking algebra for the 'subcontext' is a weak* dense subalgebra of the weak linking algebra for $(Y, X)$ (this uses 6.10 in [2; see the argument above 4.1 in 8 ).

(8) Let $Z$ be any WTRO (see (1)), and suppose that $Z^{*} Z$ is contained in a dual operator algebra $M$. Then $Y=\overline{Z M}^{w^{*}}$ is a $w^{*}$-rigged $M$-module. We call this example a $W T R O$-rigged module. We also have $\tilde{Y} \cong \overline{M Z^{*}} w^{*}$. To see all this, denote the last space by $X$, and set $N$ to be the weak* closure of $Z M Z^{*}$, a dual operator algebra containing $Z Z^{*}$. If $\left(e_{t}\right)$ is the usual approximate identity for $Z Z^{*}$ with terms of the form $\sum_{k=1}^{n} z_{k} z_{k}^{*}$, then it is routine to see that $\left(e_{t}\right)$ converges weak* to an identity $1_{N}$ for $N$. Now it is easy to check that $M, Y, X, N$ satisfy 4.4 , and we are done.

We remark that the above is a generalization of Eleftherakis' recent notion of TRO-equivalence (see e.g. [16, 17)). Indeed, a WTRO-rigged module gives a TRO-equivalence of $M$ and $N$ iff the identity $e$ of the weak* closure of $Z^{*} Z$ is $1_{M}$. For the most difficult part of this, note that if the latter holds, and if $f_{s} \rightarrow e$ weak* with $f_{s} \in Z^{*} Z$, then any $m \in M$ is an iterated weak* limit of the $f_{s} m f_{s^{\prime}}$, and it follows that $M$ equals the weak* closure of $Z^{*} N Z$.

(9) The self-dual rigged modules over a dual operator algebra $M$, considered at the start of the last section in [11, together with their unique dual space structure making $(\cdot, \cdot)$ separately weak* continuous (see [11, Lemma 5.1]) are $w^{*}$-rigged. Indeed it is easy to see from the last mentioned continuity that Definition 2.2 is satisfied.

In 9] we prove that classes (5) and (8) above coincide and also equal the $w^{*}$ rigged module direct sums of modules of the form $p_{i} M$, for projections $p_{i} \in M$. This is the analogue of a famous theorem due to Paschke (see [21] or [10, Corollary 8.5.25]). We call these projectively $w^{*}$-rigged modules. We show that they constitute a proper subclass of the $w^{*}$-rigged modules and that they have some strong properties. For example, if $Y$ is a weak* Morita equivalence $M$ - $N$-bimodule, over dual operator algebras $M$ and $N$, which is both left and right projectively $w^{*}$ rigged, then $M$ and $N$ are stably isomorphic (that is, $M_{I}(M) \cong M_{I}(N)$ completely isometrically for some cardinal $I$ ). This is a reprise of the main result of [17. 


\section{REFERENCES}

[1] M. Baillet, Y. Denizeau, and J.-F. Havet, Indice d'une espérance conditionnelle, Compositio Math. 66 (1988), 199-236. MR945550 (90e:46050)

[2] D. P. Blecher, A generalization of Hilbert modules, J. Funct. Anal. 136 (1996), 365-421. MR:1380659 (97g:46071)

[3] D. P. Blecher, Some general theory of operator algebras and their modules, pp.113-144 in "Operator Algebras and Applications", Ed. A. Katavolos, Nato ASI Series, Series C - Vol. 495, Kluwer (1997). MR1462678 (98g:46079)

[4] D. P. Blecher, A new approach to Hilbert $C^{*}$-modules, Math. Ann. 307 (1997), 253-290. MR1428873 (98d:46063)

[5] D. P. Blecher, On selfdual Hilbert modules, Operator algebras and their applications, pp. 65-80, Fields Inst. Commun., 13, Amer. Math. Soc., Providence, RI, 1997. MR 1424955 (97k:46070)

[6] D. P. Blecher, D. M. Hay, and M. Neal, Hereditary subalgebras of operator algebras, J. Operator Theory 59 (2008), 333-357. MR2411049

[7] D. P. Blecher and K. Jarosz, Isomorphisms of function modules, and generalized approximation in modulus, Trans. Amer. Math. Soc. 354 (2002), 3663-3701. MR1911516 (2003c:46064)

[8] D. P. Blecher and U. Kashyap, Morita equivalence of dual operator algebras, J. Pure and Applied Algebra 212 (2008), 2401-2412. MR2440255 (2009g:47189)

[9] D. P. Blecher and J. Kraus, On a generalization of $W^{*}$-modules, Submitted (2009).

[10] D. P. Blecher and C. Le Merdy, Operator algebras and their modules - an operator space approach, London Mathematical Society Monographs, Oxford Univ. Press, Oxford, 2004. MR2111973 (2006a:46070)

[11] D. P. Blecher and B. Magajna, Duality and operator algebras: Automatic weak ${ }^{*}$ continuity and applications, J. Funct. Anal. 224 (2005), 386-407. MR2146046 (2006g:46093)

$[12]$ D. P. Blecher, P. S. Muhly, and V. I. Paulsen, Categories of operator modules (Morita equivalence and projective modules), Mem. Amer. Math. Soc. 143 (2000), no. 681. MR1645699 (2000j:46132)

[13] Y. Denizeau and J.-F. Havet, Correspondances d'indice fini. I: Indice d'un vecteur, J. Operator Theory 32 (1994), 111-156. MR.1332446 (96e:46081)

[14] E. G. Effros, N. Ozawa, and Z.-J. Ruan, On injectivity and nuclearity for operator spaces, Duke Math. J. 110 (2001), 489-521. MR.1869114 (2002k:46151)

[15] E. G. Effros and Z.-J. Ruan, Representations of operator bimodules and their applications, J. Operator Theory 19 (1988), 137-157. MR.950830 (91e:46077)

[16] G. K. Eleftherakis, A Morita type equivalence for dual operator algebras, J. Pure Appl. Algebra 212 (2008), 1060-1071. MR2387585 (2008m:47098)

[17] G. K. Eleftherakis and V. I. Paulsen, Stably isomorphic dual operator algebras, Math. Ann. 341 (2008), 99-112. MR2377471 (2009a:46111)

[18] U. Kashyap, Morita equivalence of dual operator algebras, Ph. D. thesis (University of Houston), December 2008.

[19] U. Kashyap, A Morita theorem for dual operator algebras, J. Funct. Anal. 256 (2009), 35453567.

[20] B. Magajna, Strong operator modules and the Haagerup tensor product, Proc. London Math. Soc. 74 (1997), 201-240. MR.1416731 (98e:46072)

[21] W. L. Paschke, Inner product modules over B*-algebras, Trans. Amer. Math. Soc. 182 (1973), 443-468. MR0355613 (50:8087)

[22] M. A. Rieffel, Morita equivalence for $C^{*}$-algebras and $W^{*}$-algebras, J. Pure Appl. Algebra 5 (1974), 51-96. MR0367670(51:3912)

[23] H. Zettl, A characterization of ternary rings of operators, Adv. Math. 48 (1983), 117-143. MR700979 (84h:46093)

Department of Mathematics, University of Houston, Houston, Texas 77204-3008

E-mail address: dblecher@math.uh.edu

Department of Mathematics and Computer Science, The Citadel, 171 Moultrie Street, Charleston, South Carolina 29409

E-mail address: ukashyap1@citadel.edu 